\title{
Vitamin C supplementation reduces expression of circulating miR-451a in subjects with poorly controlled type 2 diabetes mellitus and high oxidative stress
}

\author{
Laongthip Ruknarong ${ }^{1,2,3}$, Chongchira Boonthongkaew ${ }^{2,3}$, Nisa Chuangchot ${ }^{1,2,3}$, Amonrat Jumnainsong ${ }^{1,4}$, \\ Naruemon Leelayuwat ${ }^{3,5}$, Apinya Jusakul ${ }^{1,4}$, Silvana Gaudieri ${ }^{6,7,8}$, Chanvit Leelayuwat ${ }^{\text {Corresp. 1,4 }}$ \\ ${ }^{1}$ Centre for Research and Development of Medical Diagnostic Laboratories (CMDL), Faculty of Associated Medical Sciences, Khon Kaen University, Khon \\ Kaen, Khon kean, Thailand \\ 2 Biomedical Sciences Program, Graduate School, Khon Kaen University, Khon Kaen, Thailand \\ 3 Exercise and Sport Sciences Development and Research Group (ESRG), Faculty of Medicine, Khon Kaen University, Khon Kaen, Thailand \\ 4 Department of Clinical Immunology and Transfusion Sciences, Faculty of Associated Medical Sciences, Khon Kaen University, Khon Kaen, Thailand \\ 5 Department of Physiology, Faculty of Medicine, Khon Kaen University, Khon Kaen, Thailand \\ 6 School of Human Sciences, University of Western Australia, Perth, Western Australia, Australia \\ 7 Institute for Immunology and Infectious Diseases, Murdoch University, Perth, Western Australia, Australia \\ 8 Division of Infectious Diseases, Department of Medicine, Vanderbilt University Medical Center, Vanderbilt University, Nashville, Tennessee, United States \\ Corresponding Author: Chanvit Leelayuwat \\ Email address: chanvit@kku.ac.th
}

Background: Vitamin C is an essential element required for normal metabolic function. We investigated the effect of vitamin $\mathrm{C}$ supplementation on circulating miRNA (miR) expression in subjects with poorly controlled type 2 diabetes mellitus (T2DM). Changes in miR expression were also correlated with clinical measures of disease. Methods: Pre- and post-vitamin $C$ supplementation samples from five participants who had increased vitamin $C$ levels, improved oxidative status and polymorphonuclear (PMN) function after receiving $1,000 \mathrm{mg}$ of vitamin $\mathrm{C}$ daily for six weeks were screened for miRNA expression using the NanoString miRNA assay. Differences in miRNA expression identified from the miRNA screen were validated by qRT-PCR. Results: Four miRNAs showed significantly different expression post-vitamin $C$ supplementation relative to baseline, including the downregulation of miR-451a ( -1.72 fold change $(F C), p=0.036)$ and up-regulation of miR-1253 (0.62 FC, $p=0.027)$, miR-1290 (0.53 FC, $p=0.036)$ and miR-644a ( $0.5 F C, p=0.042)$. The validation study showed only miR-451a expression was significantly different from baseline with vitamin C supplementation. MiR-451a expression was negatively correlated with vitamin C levels ( $r=-0.497, p=0.049$ ) but positively correlated with levels of malondialdehyde (MDA) $(r=0.584, p=0.017)$, cholesterol $(r=0.564, p=0.022)$ and lowdensity lipoproteins ( $L D L)(r=0.522, p=0.037)$. Bioinformatics analysis of the putative target genes of miR-451a indicated gene functions related to signaling pathways involved in cellular processes, such as the mammalian target of rapamycin (mTOR) signaling 
pathway. Conclusions: Vitamin C supplementation altered circulating miR-451a expression. The results from this pilot study suggest that miRNAs could be used as biomarkers to indicate oxidative status in subjects with T2DM and with poor glycemic control and could lead to a novel molecular strategy to reduce oxidative stress in T2DM. 
1 Vitamin C supplementation reduces expression of

2 circulating miR-451a in subjects with poorly

3 controlled type 2 diabetes mellitus and high oxidative

4 stress

Laongthip Ruknarong ${ }^{1,2,3}$, Chongchira Boonthongkaew ${ }^{2,3}$, Nisa Chuangchot ${ }^{1,2,3}$, Amonrat Jumnainsong ${ }^{1,4}$, Naruemon Leelayuwat ${ }^{3,5}$, Apinya Jusakul ${ }^{1,4}$, Silvana Gaudieri ${ }^{6,7,8}$ and Chanvit Leelayuwat ${ }^{1,4}$

${ }^{1}$ Centre for Research and Development of Medical Diagnostic Laboratories (CMDL), Faculty of Associated Medical Sciences, Khon Kaen University, Khon Kaen, Thailand.

${ }^{2}$ Biomedical Sciences Program, Graduate School, Khon Kaen University, Khon Kaen, Thailand.

${ }^{3}$ Exercise and Sport Sciences Development and Research Group (ESRG), Faculty of Medicine, Khon Kaen University, Khon Kaen, Thailand.

${ }^{4}$ Department of Clinical Immunology and Transfusion Sciences, Faculty of Associated Medical Sciences, Khon Kaen University, Khon Kaen, Thailand.

${ }^{5}$ Department of Physiology, Faculty of Medicine, Khon Kaen University, Khon Kaen, Thailand. ${ }^{6}$ School of Human Sciences, University of Western Australia, Perth, WA, Australia

${ }^{7}$ Institute for Immunology and Infectious Diseases, Murdoch University, Perth, WA, Australia

8 Division of Infectious Diseases, Department of Medicine, Vanderbilt University Medical Center, Vanderbilt University, Nashville, Tennessee, United States

\section{Corresponding Author:}

Chanvit Leelayuwat ${ }^{1,4}$

Faculty of Associated Medical Sciences, Khon Kaen University, Khon Kaen, 40002, Thailand Email address: chanvit@kku.ac.th

\section{Abstract}

Background: Vitamin $\mathrm{C}$ is an essential element required for normal metabolic function. We investigated the effect of vitamin $\mathrm{C}$ supplementation on circulating miRNA (miR) expression in subjects with poorly controlled type 2 diabetes mellitus (T2DM). Changes in miR expression were also correlated with clinical measures of disease.

Methods: Pre- and post-vitamin C supplementation samples from five participants who had increased vitamin $\mathrm{C}$ levels, improved oxidative status and polymorphonuclear (PMN) function after receiving 1,000 $\mathrm{mg}$ of vitamin $\mathrm{C}$ daily for six weeks were screened for miRNA expression using the NanoString miRNA assay. Differences in miRNA expression identified from the miRNA screen were validated by qRT-PCR. 
38 Results: Four miRNAs showed significantly different expression post-vitamin C

39

40

41

42

43

44

45

46

47

48

49

50

51

52

53

54

55

56

57

58

59

60

61

62

63

64

65

66

67

68

69

70

71

72

73

74

75

76

77

supplementation relative to baseline, including the down-regulation of miR-451a (-1.72 fold change (FC), $p=0.036)$ and up-regulation of miR-1253 (0.62 FC, p=0.027), miR-1290 (0.53 FC, $\mathrm{p}=0.036)$ and miR-644a (0.5 FC, $\mathrm{p}=0.042)$. The validation study showed only miR-451a expression was significantly different from baseline with vitamin $\mathrm{C}$ supplementation. MiR-451a expression was negatively correlated with vitamin $\mathrm{C}$ levels $(\mathrm{r}=-0.497, \mathrm{p}=0.049)$ but positively correlated with levels of malondialdehyde (MDA) $(r=0.584, p=0.017)$, cholesterol $(r=0.564$, $\mathrm{p}=0.022)$ and low-density lipoproteins $(\mathrm{LDL})(\mathrm{r}=0.522, \mathrm{p}=0.037)$. Bioinformatics analysis of the putative target genes of miR-45 la indicated gene functions related to signaling pathways involved in cellular processes, such as the mammalian target of rapamycin (mTOR) signaling pathway.

Conclusions: Vitamin $\mathrm{C}$ supplementation altered circulating miR-451a expression. The results from this pilot study suggest that miRNAs could be used as biomarkers to indicate oxidative status in subjects with T2DM and with poor glycemic control and could lead to a novel molecular strategy to reduce oxidative stress in T2DM.

\section{Introduction}

Vitamin $\mathrm{C}$, or ascorbic acid, is well known as an antioxidant required for normal metabolic function of the body and is associated with a wide spectrum of biological processes (Mandl et al. 2009). As an indication of the protective properties of vitamin $C$, studies have shown that vitamin $\mathrm{C}$ can diminish the detrimental effects of hyperglycemia such as oxidative stress, inflammation as well as endothelial dysfunction (Ceriello et al. 2013). Several studies have also reported a beneficial effect of vitamin C supplementation in type 2 diabetes mellitus (T2DM); a chronic metabolic disease associated with chronic hyperglycemia and excess free fatty acids (FFAs) (Hawkins et al. 2003). This complex disorder is characterized by insulin resistance in which the pancreas produces insufficient insulin or there is an ineffective response (American Diabetes 2018). The pathophysiology of T2DM is not fully understood but high oxidative stress and low-grade inflammation are thought to be involved and can exacerbate the development and progression of the disease. Overproduction of reactive oxygen species (ROS) during the course of T2DM can cause DNA and protein damage, lipid peroxidation, cellular and vascular dysfunction leading to diabetic complications that have been associated with high levels of oxidative stress, as indicated by the markers malondialdehyde (MDA) and $\mathrm{F}_{2}$-Isoprostanes ( $\mathrm{F}_{2} \mathrm{IsoPs}$ ), and low levels of vitamin C (Bhatia et al. 2003; Johansen et al. 2005; Kaviarasan et al. 2009). Moreover, impaired immune responses, such as neutrophil function, have been reported in subjects with T2DM (Chanchamroen et al. 2009).

There is evidence that daily supplementation with $1000 \mathrm{mg}$ /day of vitamin $\mathrm{C}$ for six weeks can improve blood glucose, lipid profiles, glycated haemoglobin (HbA1C) and insulin in subjects with T2DM (Afkhami-Ardekani \& Shojaoddiny-Ardekani 2007). The effect of vitamin C on subjects can be observed at the gene expression level and mechanistically may involve epigenetic regulation including via microRNAs (miRNAs or miRs) (Kolhe et al. 2018).

Peer) reviewing PDF | (2020:07:50778:3:0:NEW 21 Dec 2020) 
78 Supporting this model, Kim et al revealed dietary consumption of high dose vitamin $\mathrm{C}$ enhances

79 anti-oxidation and anti-glycation as well as reduction of inflammation-related miRNAs in

80 lipoproteins (Kim et al. 2015). Furthermore, treatment of human bone marrow stem cells with

81 vitamin $\mathrm{C}$ has been shown to modulate miRNA expression in these cells (Kolhe et al. 2018).

82 These studies strongly suggest that vitamin $\mathrm{C}$ can affect miRNA expression and may be involved

83 in the pathophysiology of T2DM.

84 MiRNAs are functional non-coding small RNAs typically 18-22 nucleotides (nt) in length that

85 have been identified as important regulators of gene expression. These RNA molecules mainly

86 act by repressing mRNA translation at the 3' untranslated region (3'UTR) of the target mRNA

87 and can function in cell-cell communication (Bartel 2004; Turchinovich et al. 2016). One of the

88 important properties of these small RNAs is that they are highly stable in various sample types

89 such as serum or plasma and, accordingly, can be used as a biomarker or as a target for

90 therapeutics for several diseases including T2DM, in which miRNA deregulation has been

91 associated with progression of the disease (He et al. 2017; Jimenez-Lucena et al. 2018; Maqbool

92 \& Ul Hussain 2014; Regazzi 2018). However, less is known about the effect of vitamin C

93 supplementation on miRNAs in subjects with T2DM, especially for those subjects with poor

94 glycemic control. In such cases, the high oxidative stress and low levels of plasma vitamin C

95 found in these subjects may be influenced by miRNAs and vice versa.

96 To determine the effect of vitamin $\mathrm{C}$ supplementation on miRNA expression in subjects with

97 T2DM, we investigated circulating miRNAs before and after vitamin $\mathrm{C}$ supplementation using a

98 miRNA screen. We restricted the analysis to those subjects that showed changes in plasma

99 vitamin C levels, oxidative stress and polymorphonuclear (PMN) function following vitamin C

100 supplementation. We hypothesized that the expression level of miRNAs may be affected by

101 vitamin $\mathrm{C}$ supplementation and these changes may correlate with clinical measures known to

102 reflect the pathophysiological processes in T2DM. Such associations may provide greater

103 insights into the molecular mechanisms involved in these biological processes and may be used

104 as biomarkers for responses to the dietary treatment of the disease leading to novel strategies in

105 molecular targeting of relevant genes in T2DM.

106

107

108

109 Study design and participants

110 Archived plasma samples from a subset of participants who had received vitamin C as part of a

111 study examining vitamin C supplementation in subjects with T2DM (Chuangchot et al. 2020)

112 were utilized in this study and their usage was approved by the Human Research Ethics

113 Committee of Khon Kaen University in accordance with the 1964 Declaration of Helsinki

114 (HE571264). De-identified diagnostic measures of T2DM and clinical data of the subjects were

115 received from Srinagarind Hospital, Faculty of Medicine, Khon Kaen, Thailand. The selection

116 criteria for subjects in the larger study included age between 30 and 60 years old, diagnosed as

117 having T2DM according to type 2 diabetes diagnostic criteria by the American Diabetes 
118 Association (ADA) for at least 12 months, poor glycemic control (defined by $\mathrm{HbAlc} \geq 8.5 \%$ or $11969.4 \mathrm{mmol} / \mathrm{mol}$ ) without diabetic complications, treated with oral diabetic drugs only (Metformin 120 and/or Glibenclamide), sedentary and lack of any regular exercise program for at least 6 months,

121

122

123

124

125

126

127

128

129

130

131

132

133

134

135

136

137

138

139

140

141

142

143

144

145

146

147

148

149

150

151

152

153

154

155

156

157

and living in the Khon Kaen Province, Thailand. Some subjects were treated with lipid-lowering drugs and/or anti-hypertensive drugs for the duration of the study to maintain blood pressure at $\leq$ $140 / 90 \mathrm{mmHg}$. Subjects with any other diagnosed chronic disease including neuromuscular disorder, liver and kidney disease, positive chronic infection including human immunodeficiency virus, hepatitis and tuberculosis, abnormal resting electrocardiogram, changes in medical treatment during the study period, insulin injection, blood pressure higher than 140/90 $\mathrm{mm} \mathrm{Hg}$, and smokers were excluded from the study.

The participants received $1,000 \mathrm{mg} /$ day vitamin $\mathrm{C}$ for six weeks. Overnight fasting blood samples were collected at pre-and post-vitamin $\mathrm{C}$ supplementation. Biochemical measurements were determined as follows: HbA1c by Cobas c501 (Roche Diagnostics, Mannheim, Germany); lipid profile by Reflotron Plus (Boehringer Mannheim, Mannheim, Germany); fasting blood sugar by a glucose analyzer (YSI 2300 STAT PLUS, Champaign, IL 61822, USA); and insulin by an immunoradiometric assay kit ((MP Biomedical Germany GmbH, Eschwege, Germany). Plasma vitamin C levels were measured as previously described (Zhang et al. 2009). PMN phagocytosis and oxidative burst were determined using flow cytometry as previously reported (Chuangchot et al. 2020). Plasma MDA concentration was examined using the thiobarbituric acid reactive substances (TBARS) assay (Draper et al. 1993). $\mathrm{F}_{2}$ IsoPs concentration was determined using the direct 8-iso-Prostaglandin F2 $\alpha$ Enzyme Immunoassay kit (EIA, 8-isoPGF2 $\alpha$ kit, Cayman Chemical Co). Blood samples from subjects with the following characteristics were used in this study: increased plasma vitamin C levels; increased phagocytosis and oxidative burst; and reduced oxidative stress as indicated by MDA and $\mathrm{F}_{2} \mathrm{IsoPs}$ concentrations at the post-vitamin C supplementation timepoint compared to pre-

supplementation. Five participants were selected for the screening cohort and then an additional three participants were used for the validation cohort study $(n=8)$.

\section{miRNA extraction}

Total RNA was extracted from archived plasma using the miRNeasy Serum/Plasma kit (Qiagen, Germany) according to the manufacturer's instruction. In brief, $200 \mathrm{uL}$ of the plasma was mixed with five volumes of Qiazol and a fold of chloroform. Spike-in controls, cel-miR-39 and celmiR-254, were added for normalization and as an indicator of extraction efficiency. After absorption, $14 \mathrm{uL}$ of RNase-free water was added to the spin column to elute total RNA. MiRNA concentration was measured by a Nanophotometer (IMPLEN NanoPhotometer N60, Munich, Germany).

\section{miRNA analysis}

To examine changes in miRNA expression in response to vitamin C supplementation, a miRNA microarray screen was performed on the plasma samples from the five subjects using the 
158 NanoString platform (NanoString Technologies, Seattle, WA, USA). The miRNAscreen includes

159

160

161

162

163

164

165

166

167

168

169

170

171

172

173

174

175

176

177

178

179

180

181

182

183

184

185

186

187

188

189

190

191

192

193

194

195

196

197

800 human mature-miRNAs and expression levels can be evaluated without an amplification step, thereby reducing the risk of PCR bias. Briefly, $3 \mathrm{uL}$ of total RNA was ligated to specific miR-tags and hybridized to a color-coded probe. Counting of miRNA species and digital analysis of the resulting counts were performed using the nCounter human v3 miRNA expression platform. A conservative background threshold of 100 counts was set to remove targets with low expression levels from further analysis. Six positive controls and cel-miR-254 were used for data normalization.

\section{qRT-PCR}

Quantitative real-time polymerase chain reaction (qRT-PCR) was performed to validate miRNA expression levels for a select set of miRNAs in eight subjects with T2DM. The small RNAs were converted to cDNA using the miScript II RT kit (Qiagen, Germany) following the manufacturer's protocol. The miScript primer assay and the miScript SYBR green PCR kit from Qiagen were used to measure the specific miRNAs on a Bio-Rad CF96 ${ }^{\mathrm{TM}}$ real time PCR machine (Bio-Rad, CA, USA) according to the manufacturer's protocols. The cel-miR-39 spikein control was used as a reference gene. Ct values $>35$ were deemed to be below background levels. Relative expression of individual miRNAs was calculated by the $2^{-\Delta \mathrm{Ct}}$ method.

\section{Target gene prediction and pathway analysis}

To identify the target genes of miR-451a, two bioinformatics tools Tarbase v. 8.0 and miRTarBase v. 8.0 were used and both tools have been developed based on experimental studies. The biological functions and enrichment pathways of these target genes were analyzed using the Database for Annotation, Visualization and Integration Discovery (DAVID) v. 6.8. A false discovery rate (FDR) with an adjusted P-value $<0.05$ was set as the significance threshold.

\section{Statistical analysis}

Normal distribution was tested using the Shapiro-Wilk test. Log2 transformed data by nSolver Analysis Software v. 4.0 (NanoString Technologies) was used to analyze the expression of each miRNA with the visual genomics analysis studio (VGAS) program

(http://www.iiid.com.au/software/vgas). Comparison of the pre- and post-vitamin C supplementation data was performed using a paired t-test. Laboratory measures and values from the validation study were compared between pre-and post-supplementation by paired t-test and Wilcoxon signed ranks test using SPSS statistics v. 19 (SPSS Inc., Chicago, IL, USA). A power of test (1- $\beta$ ) was checked by post-hoc analysis using G*Power v. 3.1.9.2 (Heinrich-HeineUniversität Düsseldorf, Germany). Spearman correlation was used to analyze the correlation between circulating miRNA expression and blood parameters and the figures were generated using GraphPad Prism v. 5.0 (GraphPad Software Inc., CA, USA). A p-value of $<0.05$ was set as the significance threshold. All data expressed as mean $\pm \mathrm{SD}$. 
198

199

200

201

202

203

204

205

206

207

208

209

210

211

212

213

214

215

216

217

218

219

220

221

222

223

224

225

226

227

228

229

230

231

232

233

234

235

236

237

\section{Results}

Characteristics of subjects at pre- and post-vitamin $\mathbf{C}$ supplementation timepoints Subjects with T2DM in the current study had an average age of 59 years, BMI of $26 \mathrm{Kg} / \mathrm{m}^{2}$, $\mathrm{HbA} 1 \mathrm{c}$ of $95 \mathrm{mmol} / \mathrm{mol}$ or $10.8 \%$ and duration of disease of 9 years (Table 1). Compared to baseline pre-vitamin C supplementation levels, subjects showed significantly increased plasma vitamin $\mathrm{C}$ levels, raised PMN phagocytosis and oxidative burst, reduced products of lipid peroxidase (MDA and $\mathrm{F}_{2} \mathrm{IsoPs}$ ), as well as reduced levels of cholesterol following daily vitamin C supplementation for six weeks (Table 1).

\section{MiRNA screen revealed differences in the expression level of a subset of miRNAs at pre-} and post-vitamin $C$ supplementation

A screen of circulating miRNAs in the plasma of five subjects with T2DM at pre- and postsupplementation was performed using the NanoString platform. Of the 800 target miRNA in the screen, 26 miRNAs were expressed in all samples (Supplementary Fig. S1 and Table S1).

Compared to pre-supplementation levels, four miRNAs showed significantly different expression at post-supplementation. Of these, miR-451a was down-regulated post-supplementation (-1.72 fold change (FC), $\mathrm{p}=0.036$ ), while miR-1253 (0.62 FC, $\mathrm{p}=0.027)$, miR-1290 (0.53 FC, $\mathrm{p}=0.036)$ and miR-644a ( $0.5 \mathrm{FC}, \mathrm{p}=0.042)$ were up-regulated post-supplementation (Fig. 1A). MiR-451a showed the highest reduced fold change of any of the other miRNAs tested. To validate these differences, samples from three additional subjects with T2DM were included in the qPCR analysis. Only miR-451a expression was found to be significantly different post-supplementation compared to the pre-supplementation timepoint ( $\mathrm{p}=0.030$ ) (Fig. 1B). Using the same approach as above, we have recently demonstrated that there is no difference in the level of miR-451a in the plasma of placebo controls pre- and post-vitamin C supplementation (Chuangchot et al. 2020) (Supplementary Fig. S2).

\section{Correlation between expression of circulating miRNA and clinical laboratory measures of T2DM}

The expression of miR-451a was then correlated with clinical laboratory measures for the subjects with T2DM and included all data from both the pre-and post-vitamin C supplementation timepoints. Expression of miR-451a was negatively correlated with plasma vitamin $\mathrm{C}$ levels ( $\mathrm{r}=-$ $0.497, \mathrm{p}=0.049$, Fig. $2 \mathrm{~A}$ ) but positively correlated with MDA ( $\mathrm{r}=0.584, \mathrm{p}=0.017$, Fig. $2 \mathrm{~B})$, cholesterol ( $r=0.564, p=0.022$, Fig. $2 \mathrm{C}$ ) and LDL ( $r=0.522$, $\mathrm{p}=0.037$, Fig. 2D) levels, suggesting that this miRNA might play a role in oxidative status and lipid metabolism.

\section{Target gene prediction and pathway enrichment analysis}

Bioinformatics analysis tools were used to identify putative target genes and pathways likely to be affected by miR-451a. A total of 88 target genes of miR-451a were retrieved from the analysis and used in the program DAVID to identify relevant KEGG pathways. The top enriched 
238 KEGG pathways are presented in Table 2. The target genes are involved in several signaling 239 pathways including mTOR signaling pathway, PI3K-Akt signaling pathway, estrogen signaling 240 pathway, hepatitis B, pathway in cancer and colorectal cancer, AMPK signaling pathway, FoxO 241 signaling pathway as well as other signaling pathways that share target genes such as IKBKB, 242 PIK3CA and AKT1 as shown in Table 2 (description of genes in Supplementary Table S2).

243

\section{Discussion}

245 Type 2 diabetes mellitus (T2DM) is a worldwide health problem and continues to increase in 246 prevalence (Cho et al. 2018). Overproduction of ROS caused by high glucose levels and 247 dyslipidemia in long-standing T2DM leads to increased oxidative stress and chronic 248 inflammation. These features can result in diabetic complications and increased susceptibility to infections resulting in increased morbidity and mortality, especially in uncontrolled diabetes (Critchley et al. 2018). These pathophysiological processes could impact miRNA expression and as suggested by numerous studies, which have investigated several dysregulated miRNAs in T2DM, these molecules can be used as potential biomarkers for predictive, diagnostic and therapeutic procedures (He et al. 2017; Jimenez-Lucena et al. 2018). Furthermore, there is evidence that upregulation of miR-21 is associated with elevated ROS levels and reduced antioxidant responses in prediabetics and subjects with T2DM (La Sala et al. 2019), indicating miRNA may be linked with pathological processes of the disease. Interestingly, vitamin C supplementation in subjects with T2DM has shown positive effects on blood glucose and oxidative stress (Afkhami-Ardekani \& Shojaoddiny-Ardekani 2007; Mazloom et al. 2011). We hypothesized that vitamin C supplementation could modulate miRNA expression and correlate with the blood parameters of subjects for which we observed improved plasma vitamin $\mathrm{C}$ levels, reduced oxidative stress and increased PMN function after receiving 1,000 $\mathrm{mg}$ of vitamin $\mathrm{C}$ daily for six weeks. To our knowledge, the present study is the first report to investigate the effect of vitamin C supplementation on miRNA expression in subjects with T2DM and with poor glycemic control. The results from the initial miRNA screen revealed that miR-451a was downregulated, with the highest negative fold change, after supplementation and this was validated using qRT-PCR. However, the three significantly upregulated miRNAs from the microarray screen did not show differences in expression pre- and post-supplementation in the qRT-PCR validation step that also utilized additional samples. The discrepancy between the two platforms likely reflects the low template of these miRNAs and as such we focused only on miR-451a for further analysis. MiR-451a (or miR-451, miRBase 22) is dicer independent and can be transcribed into a hairpin structure and then processed with the Argonaute 2 (Ago 2) protein (Wang et al. 2019). This miRNA is stable in peripheral blood and can be found in erythrocytes, PMN, mononuclear cells and platelets (Ghai et al. 2019; Masaki et al. 2007). MiR-451a has been reported in the blood circulation of elderly subjects with T2DM and is up-regulated in plasma samples of subjects with diabetic nephropathy as well as in the serum of subjects with acute diabetic Charcot foot, indicating the expressed miRNA may play a role in pathological processes of the disease 
278 (Catanzaro et al. 2018; Pasquier et al. 2018; Sayilar et al. 2016). In this study, the expression of 279 miR-451a was negatively correlated with vitamin C levels but positively correlated with MDA 280 levels, a marker of oxidative stress, suggesting a possible role in the oxidative status of the 281 subjects.

282 MiR-451a is also important in the erythroid lineage and plays a role in ROS production in 283 erythrocytes by targeting 14-3-3zeta via the inhibition of FoxO3 (Yu et al. 2010). High oxidative 284 stress may affect miR-451a production as a study from Ranjan et al found that deficient miR285 451a expression was associated with defective ROS generation due to reduced Ago2 protein 286 levels in macrophages (Ranjan et al. 2015). Inhibition of miR-451a also reduced ROS, lipid 287 peroxidation and DNA damage (Zhu et al. 2018). These data suggest that miR-451a expression 288 may positively correlate with ROS production. In this study, miR-451a expression showed a 289 positive correlation with cholesterol and LDL. These factors may exacerbate the progression of 290 the disease accompanied with miR-451a expression. However, further work is needed to confirm 291 the correlation of miR-451a with oxidative stress as found in the current study.

292 We also investigated the target genes of miR-451a and their functional enrichment using 293 experimentally validated datasets. The target genes were significantly involved in signaling 294 pathways that play an important role in cellular functions of biological processes as shown in 295 Fig. 3 and Table 2. To support our results, studies in T2DM cardiomyopathy-induced mice with 296 high ROS production displayed up-regulation of miR-451a that directly targeted calcium-binding 297 protein 39 (CAB39) and resulted in down-regulation of the LKB1/AMPK signaling pathway. 298 These target genes are listed in Table 2. Furthermore, knockout of this miRNA showed the 299 opposite effect (Kuwabara et al. 2015). Thus, the data suggests that miR-451a expression was 300 induced by high oxidative stress leading to suppression of the AMPK pathway, a central energy301 sensing of metabolic regulation, which was found to have reduced activity in insulin-resistant 302 individuals (Li et al. 2019; Xu et al. 2012). The reduced ROS production found in the subjects 303 with T2DM in this study after vitamin C supplementation and with down-regulation of miR-451a 304 may be through CAB39/LKB1/AMPK signaling, as PI3K/AKT/mTOR signaling is the central 305 pathway of glucose and lipid metabolism (Huang et al. 2018; Tuo \& Xiang 2018).

306 Hyperglycemia and excess FFAs lead to insulin resistance and high levels of ROS resulting in 307 impaired PI3K/AKT/mTOR signaling found in subjects with T2DM (Huang et al. 2018).

308 Moreover, reduction of oxidative stress has been reported in subjects with up-regulation of AKT 309 and mTOR after pretreatment with vitamin C (Lin et al. 2016). MiR-451a could target multiple 310 genes involved in the PI3K/AKT/mTOR signaling pathway including AKT1, PIK3CA, PIK3R1, 311 and PRKAA1 (as indicated in Table 2 and Supplementary Table S2). In addition, IKBKB is one 312 of the target genes of miR-451a ( $L i$ et al. 2013) and is a known inhibitor of the NF-kB complex 313 that can be activated by multiple harmful cellular stimuli such as stress or free radicals. There is 314 evidence that vitamin $\mathrm{C}$ can also diminish NF-kB activation by directly inhibiting IKK $\beta$, aside 315 from quenching ROS (Carcamo et al. 2004). The miRNA may be involved in this synergistic 316 effect of vitamin C in response to ROS. Supplementation with vitamin $\mathrm{C}$ in the subjects might 317 help to promote these pathways through the regulation of miR-451a. Further studies are required 
318 to explore the mechanisms behind the effects of vitamin $\mathrm{C}$ supplementation on this circulating 319 miRNA associated with systemic oxidative stress in the subjects.

320 The participants in this study showed enhanced phagocytosis and oxidative burst after

321 supplementation of vitamin $\mathrm{C}$, which is consistent with previous studies that have shown the

322 effect of vitamin $\mathrm{C}$ in enhancing PMN function such as motility, chemotaxis, phagocytosis and

323 microbial killing (Bozonet et al. 2015; Carr \& Maggini 2017). However, knowledge of the

324

325

326

327

328

329

330

331

332

333

334

335

336

337

338

339

340

341

342

343

344

345

346

347

348

349

350

351

352

353

354

355

356

357 molecular mechanisms of the effect of vitamin $\mathrm{C}$ on these processes, especially phagocytosis and oxidative burst, are limited. MiR-451 a has been reported to impact neutrophil chemotaxis by suppressing p38 MAPK through targeting Rab5a and 14-3-3zeta (Murata et al. 2014). Rab5a, a small GTPase, regulates intracellular membrane trafficking involved in phagolysosome fusion during bacteria phagocytosis of neutrophils (Perskvist et al. 2002). Moreover, treatment of human bladder cancer cells by vitamin $\mathrm{C}$ could suppress p 38 MAPK activity and ROS production (Kim et al. 2008). As suggested from these data, vitamin C may affect miR-451a expression and associate with neutrophil function through targeting Rab5a and the p38 MAPK pathway. However, investigation of intracellular miRNAs in response to vitamin C supplementation may help us to better understand the molecular mechanism underlying PMN function that we clearly observed in these subjects. A proposed mechanism of action for miR451a in response to vitamin $\mathrm{C}$ supplementation is shown in Fig. 3.

Limitations of this pilot study include a small sample size. Increasing the number of the participants may better clarify the association of the candidate miRNA and the laboratory measures including improved oxidative status and PMN functions as well as reduced cholesterol after vitamin C supplementation. Nevertheless, the power of the test based on the difference between the two dependent groups using the mean and S.D. of relative miR-451a data revealed that the effect size was 1.15 and the power of the test was approximated at $80 \%$. Furthermore, the recent study compared only the miRNA expression between the pre-and post-vitamin C supplementation. However, our previous study of subjects receiving placebo showed no difference in miR-451a expression at pre-and post-supplementation as represented in Supplementary Fig. S2. More research is needed to confirm and validate the finding that vitamin $\mathrm{C}$ intake modulates circulating miR-451a expression and to determine the functional role of this miRNA in subjects with uncontrolled T2DM.

\section{Conclusions}

In conclusion, this study has identified a novel association between vitamin $\mathrm{C}$ supplementation for six weeks and reduced circulating miR-451a expression in subjects with poorly controlled T2DM. This candidate miRNA may be used as a biomarker to identify subjects that respond to vitamin $\mathrm{C}$ treatment or oxidative status in plasma.

\section{Acknowledgements}

We would like to thank Exercise and Sport Sciences Development and Research Group (ESRG), Khon Kaen University for laboratory data collection used in this work. 
358

359

360

361

362

363

364

365

366

367

368

369

370

371

372

373

374

375

376

377

378

379

380

381

382

383

384

385

386

387

388

389

390

391

392

393

394

395

396

397

398

\section{ADDITIONAL INFORMATION AND DECLARATIONS}

\section{Funding}

This work was supported by The Royal Golden Jubilee (RGJ) Ph.D. Programme

(PHD/0045/2557), The Centre for Research and Development of Medical Diagnostic

Laboratories (CMDL), Faculty of Associated Medical Sciences, Khon Kaen University, Exercise and Sport Sciences Development and Research Group, and Faculty of Medicine, Khon Kaen University.

\section{Competing Interests}

The authors declare no competing interests.

\section{Author contributions}

Laongthip Ruknarong performed experiments, analysed the data, prepared figures and tables, prepared the first draft manuscript and the final draft.

Chongchira Boonthongkaew and Nisa Chuangchot enrolled participants, performed experiments and analysed the data

Amonrat Jumnainsong, Naruemon Leelayuwat and Apinya Jusakul reviewed and approved the final draft.

Silvana Gaudieri and Chanvit Leelayuwat analysed, criticised the data, presentations of the manuscript, reviewed and approved the final draft.

Chanvit Leelayuwat design of the study, oversaw and supervised the whole project.

\section{References}

Afkhami-Ardekani M, and Shojaoddiny-Ardekani A. 2007. Effect of vitamin C on blood glucose, serum lipids \& serum insulin in type 2 diabetes patients. Indian J Med Res 126:471-474.

American Diabetes A. 2018. 2. Classification and Diagnosis of Diabetes: Standards of Medical Care in Diabetes-2018. Diabetes Care 41:S13-S27. 10.2337/dc18-S002

Bartel DP. 2004. MicroRNAs: genomics, biogenesis, mechanism, and function. Cell 116:281297. 10.1016/s0092-8674(04)00045-5

Bhatia S, Shukla R, Venkata Madhu S, Kaur Gambhir J, and Madhava Prabhu K. 2003. Antioxidant status, lipid peroxidation and nitric oxide end products in patients of type 2 diabetes mellitus with nephropathy. Clin Biochem 36:557-562.

Bozonet SM, Carr AC, Pullar JM, and Vissers MC. 2015. Enhanced human neutrophil vitamin $\mathrm{C}$ status, chemotaxis and oxidant generation following dietary supplementation with vitamin C-rich SunGold kiwifruit. Nutrients 7:2574-2588. 10.3390/nu7042574

Carcamo JM, Pedraza A, Borquez-Ojeda O, Zhang B, Sanchez R, and Golde DW. 2004. Vitamin $C$ is a kinase inhibitor: dehydroascorbic acid inhibits IkappaBalpha kinase beta. Mol Cell Biol 24:6645-6652. 10.1128/MCB.24.15.6645-6652.2004 
399

400

401

402

403

404

405

406

407

408

409

410

411

412

413

414

415

416

417

418

419

420

421

422

423

424

425

426

427

428

429

430

431

432

433

434

435

436

437

438

439

440

441

442

Carr AC, and Maggini S. 2017. Vitamin C and Immune Function. Nutrients 9(11):1211. 10.3390/nu9111211

Catanzaro G, Besharat ZM, Chiacchiarini M, Abballe L, Sabato C, Vacca A, Borgiani P, Dotta F, Tesauro M, Po A, and Ferretti E. 2018. Circulating MicroRNAs in Elderly Type 2 Diabetic Patients. Int J Endocrinol 2018:6872635. 10.1155/2018/6872635

Ceriello A, Novials A, Ortega E, Canivell S, Pujadas G, La Sala L, Bucciarelli L, Rondinelli M, and Genovese S. 2013. Vitamin C further improves the protective effect of GLP-1 on the ischemia-reperfusion-like effect induced by hyperglycemia post-hypoglycemia in type 1 diabetes. Cardiovasc Diabetol 12:97. 10.1186/1475-2840-12-97

Chanchamroen S, Kewcharoenwong C, Susaengrat W, Ato M, and Lertmemongkolchai G. 2009. Human polymorphonuclear neutrophil responses to Burkholderia pseudomallei in healthy and diabetic subjects. Infect Immun 77:456-463. 10.1128/IAI.00503-08

Cho NH, Shaw JE, Karuranga S, Huang Y, da Rocha Fernandes JD, Ohlrogge AW, and Malanda B. 2018. IDF Diabetes Atlas: Global estimates of diabetes prevalence for 2017 and projections for 2045. Diabetes Res Clin Pract 138:271-281.

10.1016/j.diabres.2018.02.023

Chuangchot $\mathbf{N}$, Boonthongkaew $\mathrm{C}$, Phoksawat $\mathrm{W}$, Jumnainsong A, Leelayuwat $\mathrm{C}$, and Leelayuwat N. 2020. Oral vitamin $C$ treatment increases polymorphonuclear cell functions in type 2 diabetes mellitus patients with poor glycemic control. Nutr Res 79:5059. 10.1016/j.nutres.2020.05.010

Critchley JA, Carey IM, Harris T, DeWilde S, Hosking FJ, and Cook DG. 2018. Glycemic Control and Risk of Infections Among People With Type 1 or Type 2 Diabetes in a Large Primary Care Cohort Study. Diabetes Care 41:2127-2135. 10.2337/dc18-0287

Draper HH, Squires EJ, Mahmoodi H, Wu J, Agarwal S, and Hadley M. 1993. A comparative evaluation of thiobarbituric acid methods for the determination of malondialdehyde in biological materials. Free Radic Biol Med 15:353-363. 10.1016/0891-5849(93)90035-s

Ghai V, Kim TK, Etheridge A, Nielsen T, Hansen T, Pedersen O, Galas D, and Wang K. 2019. Extracellular Vesicle Encapsulated MicroRNAs in Patients with Type 2 Diabetes Are Affected by Metformin Treatment. J Clin Med 8(5):617. 10.3390/jcm8050617

Hawkins M, Tonelli J, Kishore P, Stein D, Ragucci E, Gitig A, and Reddy K. 2003. Contribution of elevated free fatty acid levels to the lack of glucose effectiveness in type 2 diabetes. Diabetes 52:2748-2758. 10.2337/diabetes.52.11.2748

He Y, Ding Y, Liang B, Lin J, Kim TK, Yu H, Hang H, and Wang K. 2017. A Systematic Study of Dysregulated MicroRNA in Type 2 Diabetes Mellitus. Int J Mol Sci 18(3): 456. 10.3390/ijms18030456

Huang X, Liu G, Guo J, and Su Z. 2018. The PI3K/AKT pathway in obesity and type 2 diabetes. Int J Biol Sci 14:1483-1496. 10.7150/ijbs.27173

Jimenez-Lucena R, Rangel-Zuniga OA, Alcala-Diaz JF, Lopez-Moreno J, Roncero-Ramos I, Molina-Abril H, Yubero-Serrano EM, Caballero-Villarraso J, Delgado-Lista J, Castano JP, Ordovas JM, Perez-Martinez P, Camargo A, and Lopez-Miranda J. 2018. Circulating miRNAs as Predictive Biomarkers of Type 2 Diabetes Mellitus Development in Coronary Heart Disease Patients from the CORDIOPREV Study. Mol Ther Nucleic Acids 12:146-157. 10.1016/j.omtn.2018.05.002 
443

444

445

446

447

448

449

450

451

452

453

454

455

456

457

458

459

460

461

462

463

464

465

466

467

468

469

470

471

472

473

474

475

476

477

478

479

480

481

482

483

484

485

486

487

488

Johansen JS, Harris AK, Rychly DJ, and Ergul A. 2005. Oxidative stress and the use of antioxidants in diabetes: linking basic science to clinical practice. Cardiovasc Diabetol 4:5. 10.1186/1475-2840-4-5

Kaviarasan S, Muniandy S, Qvist R, and Ismail IS. 2009. F(2)-isoprostanes as novel biomarkers for type 2 diabetes: a review. J Clin Biochem Nutr 45:1-8. 10.3164/jcbn.08266

Kim JE, Jin DH, Lee SD, Hong SW, Shin JS, Lee SK, Jung DJ, Kang JS, and Lee WJ. 2008. Vitamin $C$ inhibits p53-induced replicative senescence through suppression of ROS production and p38 MAPK activity. Int J Mol Med 22:651-655.

Kim SM, Lim SM, Yoo JA, Woo MJ, and Cho KH. 2015. Consumption of high-dose vitamin $\mathrm{C}$ (1250 mg per day) enhances functional and structural properties of serum lipoprotein to improve anti-oxidant, anti-atherosclerotic, and anti-aging effects via regulation of antiinflammatory microRNA. Food Funct 6:3604-3612. 10.1039/c5fo00738k

Kolhe R, Mondal AK, Pundkar C, Periyasamy-Thandavan S, Mendhe B, Hunter M, Isales CM, Hill WD, Hamrick MW, and Fulzele S. 2018. Modulation of miRNAs by Vitamin C in Human Bone Marrow Stromal Cells. Nutrients 10(2):186.. 10.3390/nu10020186

Kuwabara Y, Horie T, Baba O, Watanabe S, Nishiga M, Usami S, Izuhara M, Nakao T, Nishino T, Otsu K, Kita T, Kimura T, and Ono K. 2015. MicroRNA-451 exacerbates lipotoxicity in cardiac myocytes and high-fat diet-induced cardiac hypertrophy in mice through suppression of the LKB1/AMPK pathway. Circ Res 116:279-288.

10.1161/CIRCRESAHA.116.304707

La Sala L, Mrakic-Sposta S, Tagliabue E, Prattichizzo F, Micheloni S, Sangalli E, Specchia C, Uccellatore AC, Lupini S, Spinetti G, de Candia P, and Ceriello A. 2019. Circulating microRNA-21 is an early predictor of ROS-mediated damage in subjects with high risk of developing diabetes and in drug-naive T2D. Cardiovasc Diabetol 18:18. 10.1186/s12933-019-0824-2

Li HP, Zeng XC, Zhang B, Long JT, Zhou B, Tan GS, Zeng WX, Chen W, and Yang JY. 2013. miR-451 inhibits cell proliferation in human hepatocellular carcinoma through direct suppression of IKK-beta. Carcinogenesis 34:2443-2451. 10.1093/carcin/bgt206

Li J, Wan W, Chen T, Tong S, Jiang X, and Liu W. 2019. miR-451 Silencing Inhibited Doxorubicin Exposure-Induced Cardiotoxicity in Mice. Biomed Res Int 2019:1528278. $10.1155 / 2019 / 1528278$

Lin CJ, Chen TL, Tseng YY, Wu GJ, Hsieh MH, Lin YW, and Chen RM. 2016. Honokiol induces autophagic cell death in malignant glioma through reactive oxygen speciesmediated regulation of the p53/PI3K/Akt/mTOR signaling pathway. Toxicol Appl Pharmacol 304:59-69. 10.1016/j.taap.2016.05.018

Mandl J, Szarka A, and Banhegyi G. 2009. Vitamin C: update on physiology and pharmacology. Br J Pharmacol 157:1097-1110. 10.1111/j.1476-5381.2009.00282.x

Maqbool R, and UI Hussain M. 2014. MicroRNAs and human diseases: diagnostic and therapeutic potential. Cell Tissue Res 358:1-15. 10.1007/s00441-013-1787-3

Masaki S, Ohtsuka R, Abe Y, Muta K, and Umemura T. 2007. Expression patterns of microRNAs 155 and 451 during normal human erythropoiesis. Biochem Biophys Res Commun 364:509-514. 10.1016/j.bbrc.2007.10.077

Mazloom Z, Hejazi N, Dabbaghmanesh MH, Tabatabaei HR, Ahmadi A, and Ansar H. 2011. Effect of vitamin $C$ supplementation on postprandial oxidative stress and lipid profile in type 2 diabetic patients. Pak J Biol Sci 14:900-904. 10.3923/pjbs.2011.900.904

PeerJ reviewing PDF | (2020:07:50778:3:0:NEW 21 Dec 2020) 
489

490

491

492

493

494

495

496

497

498

499

500

501

502

503

504

505

506

507

508

509

510

511

512

513

514

515

516

517

518

519

520

521

522

523

524

525

526

527

528

529

530

531

532

533

534

Murata K, Yoshitomi H, Furu M, Ishikawa M, Shibuya H, Ito H, and Matsuda S. 2014. MicroRNA-451 down-regulates neutrophil chemotaxis via p38 MAPK. Arthritis Rheumatol 66:549-559. 10.1002/art.38269

Pasquier J, Ramachandran V, Abu-Qaoud MR, Thomas B, Benurwar MJ, Chidiac O, Hoarau-Vechot J, Robay A, Fakhro K, Menzies RA, Jayyousi A, Zirie M, Al Suwaidi J, Malik RA, Talal TK, Najafi-Shoushtari SH, Rafii A, and Abi Khalil C. 2018. Differentially expressed circulating microRNAs in the development of acute diabetic Charcot foot. Epigenomics 10:1267-1278. 10.2217/epi-2018-0052

Perskvist N, Roberg K, Kulyte A, and Stendahl O. 2002. Rab5a GTPase regulates fusion between pathogen-containing phagosomes and cytoplasmic organelles in human neutrophils. J Cell Sci 115:1321-1330.

Ranjan R, Lee YG, Karpurapu M, Syed MA, Chung S, Deng J, Jeong JJ, Zhao G, Xiao L, Sadikot RT, Weiss MJ, Christman JW, and Park GY. 2015. p47phox and reactive oxygen species production modulate expression of microRNA-451 in macrophages. Free Radic Res 49:25-34. 10.3109/10715762.2014.974037

Regazzi R. 2018. MicroRNAs as therapeutic targets for the treatment of diabetes mellitus and its complications. Expert Opin Ther Targets 22:153-160. 10.1080/14728222.2018.1420168

Sayilar EI, Gullulu M, Tuncel E, Peynirci H, Alemdar A, Tunca B, Egeli U, Cecener G, Bayindir M, and Cosgun G. 2016. Biomarker Potential of Urine miR-451 at Different Stages of Diabetic Nephropathy. Journal of Diabetes \& Metabolism 7:2. Unsp 1000650 10.4172/2155-6156.1000650

Tuo Y, and Xiang M. 2018. mTOR: A double-edged sword for diabetes. J Leukoc Biol 106(2):385-395. 10.1002/JLB.3MR0317-095RR

Turchinovich A, Tonevitsky AG, and Burwinkel B. 2016. Extracellular miRNA: A Collision of Two Paradigms. Trends Biochem Sci 41:883-892. 10.1016/j.tibs.2016.08.004

Wang T, Wu F, and Yu D. 2019. miR-144/451 in hematopoiesis and beyond. ExRNA 1:16. 10.1186/s41544-019-0035-8

Xu XJ, Gauthier MS, Hess DT, Apovian CM, Cacicedo JM, Gokce N, Farb M, Valentine RJ, and Ruderman NB. 2012. Insulin sensitive and resistant obesity in humans: AMPK activity, oxidative stress, and depot-specific changes in gene expression in adipose tissue. J Lipid Res 53:792-801. 10.1194/j1r.P022905

Yu D, dos Santos CO, Zhao G, Jiang J, Amigo JD, Khandros E, Dore LC, Yao Y, D'Souza J, Zhang Z, Ghaffari S, Choi J, Friend S, Tong W, Orange JS, Paw BH, and Weiss MJ. 2010. miR-451 protects against erythroid oxidant stress by repressing 14-3-3zeta. Genes Dev 24:1620-1633. 10.1101/gad.1942110

Zhang H, Li J, Wang K, Du X, and Li Q. 2009. A simple and sensitive assay for ascorbate using potassium ferricyanide as spectroscopic probe reagent. Anal Biochem 388:40-46. 10.1016/j.ab.2009.01.039

Zhu H, Zhang L, Xu J, Zhu C, Zhao H, Zhu Y, and Lv G. 2018. AntogomiR-451 protects human gastric epithelial cells from ethanol via activating AMPK signaling. Biochem Biophys Res Commun 497:339-346. 10.1016/j.bbrc.2018.02.082

\section{Figure legends}

Figure 1 Patterns of miRNA expression. (A) Analysis of circulating miRNA expression combined with clinical measures and qRT-PCR data in response to vitamin $\mathrm{C}$ supplementation in T2DM subjects with poor glycemic control $(n=5)$. For the miRNA, black and red bubbles

Peer] reviewing PDF | (2020:07:50778:3:0:NEW 21 Dec 2020) 
535 represent pre-and post-supplementation, respectively. The size of the bubbles reflects the count 536 of the specific miRNA relative to other miRNAs. The laboratory measures and miR-451a qRT-

537 PCR data are indicated as a heat map from minimum value to maximum value of each parameter 538 independently of other parameters; yellow to blue represents the low to high range. Pre; pre539 vitamin C, Post; post-vitamin C supplementation. Numbers represent subjects 1-5. (B) Plot 540 represents relative expression of miR-451a as validated by qRT-PCR $(\mathrm{n}=8)$.

541

542 Figure 2 Correlation between miR-451a expression and laboratory variables. (A) Vitamin C

543 levels, (B) MDA, (C) Cholesterol and (D) LDL. Black and red represent pre-and post-

544 supplementation samples, respectively.

545

546

Figure 3 Proposed mechanism of the effect of miR-451a in response to vitamin C

547 supplementation. Red lines represent the results of this study and blue lines indicate potential

548 mode of interaction for miR-451a. Poor glycemic controlled T2DM subjects receiving vitamin C

$5491,000 \mathrm{mg}$ daily for six weeks showed significant down-regulation of circulating miR-451a

550 accompanied with increased levels of vitamin $\mathrm{C}$, reduced oxidative stress (MDA and $\mathrm{F}_{2} \mathrm{IsoPs}$ )

551 and increased PMN function (phagocytosis and oxidative burst). Changes in oxidative status may

552 modulate miR-451a expression by altering Ago2 protein and/or increased involvement of

553 signaling pathways such as AMPK signaling by repressing its target genes. MiR-451a may play

554 a role in neutrophil chemotaxis by targeting RAB5A and 14-3-3zeta resulting in the activation of

555 p38 MAPK signaling. However, the role of this miRNA in phagocytosis and oxidative burst of

556 neutrophils is still to be explored (dotted line) and may be reflected in intracellular miRNA

557 changes rather than circulating changes as identified in this study. 


\section{Table 1 (on next page)}

Table 1 : Characteristics of the participants at the pre-and post-vitamin C supplementation timepoints. 
1 Table 1: Characteristics of the participants at the pre-and post-vitamin $\mathrm{C}$ supplementation

2 timepoints.

3

\begin{tabular}{|c|c|c|c|}
\hline Parameters & $\begin{array}{l}\text { Pre- } \\
\text { supplementation } \\
(n=8)\end{array}$ & $\begin{array}{l}\text { Post- } \\
\text { supplementation } \\
(n=8)\end{array}$ & P-value \\
\hline Gender (female/male) & $7 / 1$ & $7 / 1$ & - \\
\hline Age (year) & $58.8 \pm 5.9$ & $58.8 \pm 5.9$ & - \\
\hline Duration of the disease (year) & $9.3 \pm 6.6$ & $9.3 \pm 6.6$ & - \\
\hline $\operatorname{BMI}\left(\mathrm{Kg} / \mathrm{m}^{2}\right)$ & $26.2 \pm 4.1$ & $26.2 \pm 3.9$ & 0.861 \\
\hline $\mathrm{HbA} 1 \mathrm{c}(\mathrm{mmol} / \mathrm{mol})$ & $95.0 \pm 15.0$ & $95.0 \pm 14.8$ & 0.909 \\
\hline FBS $(\mathrm{mmol} / \mathrm{L})$ & $13.0 \pm 4.3$ & $10.0 \pm 2.0$ & 0.059 \\
\hline Plasma ascorbate $(\mu \mathrm{mol} / \mathrm{L})$ & $57.8 \pm 11.0$ & $90.5 \pm 55.5$ & 0.017 \\
\hline Plasma MDA levels $(\mu \mathrm{mol} / \mathrm{mL})$ & $17.0 \pm 8.6$ & $10.6 \pm 3.8$ & 0.031 \\
\hline Plasma $F_{2}$ IsoPs (pg/mL) & $16.9 \pm 4.8$ & $12.0 \pm 4.3$ & 0.012 \\
\hline Phagocytosis $(\%)$ & $20.4 \pm 8.6$ & $30.7 \pm 11.4$ & 0.012 \\
\hline Oxidative burst (\%) & $5.9 \pm 3.5$ & $11.1 \pm 4.5$ & 0.006 \\
\hline Cholesterol (mg/dL) & $239.3 \pm 60.1$ & $187.9 \pm 45.9$ & 0.050 \\
\hline Triglyceride (mg/dL) & $168.8 \pm 63.8$ & $195.9 \pm 100.1$ & 0.217 \\
\hline $\mathrm{HDL}(\mathrm{mg} / \mathrm{dL})$ & $44.9 \pm 10.6$ & $45.9 \pm 15.0$ & 0.735 \\
\hline LDL (mg/dL) & $164.9 \pm 53.7$ & $126.5 \pm 32.3$ & 0.154 \\
\hline Insulin (IU/mL) & $14.1 \pm 4.6$ & $11.5 \pm 3.6$ & 0.057 \\
\hline
\end{tabular}

4 Data represented as mean \pm S.D. P-value was calculated using paired t-test.

5 Abbreviations: BMI, body mass index; $\mathrm{F}_{2} \mathrm{IsoPs}, \mathrm{F}_{2}$-Isoprostanes; FBS, fasting blood sugar;

6 HbA1c, glycated haemoglobin; HDL, high-density lipoproteins; LDL, low- density lipoproteins

7 MDA, malondialdehyde. 
Table 2 (on next page)

Table 2: KEGG signaling pathways of miR-451a. 
1 Table 2: KEGG signaling pathways of miR-451a.

2

\begin{tabular}{|c|c|c|c|}
\hline Term & Gene targets & P-value & FDR \\
\hline mTOR signaling pathway & $\begin{array}{l}\text { IKBKB, STK11, PRKAA1, CAB39, } \\
\text { PIK3CA, AKT1, BRAF, PIK3R1 }\end{array}$ & $1.35 \mathrm{E}-08$ & $1.13 \mathrm{E}-06$ \\
\hline $\begin{array}{l}\text { PI3K-Akt signaling } \\
\text { pathway }\end{array}$ & $\begin{array}{l}\text { IKBKB, ATF2, STK11, PRKAA1, } \\
\text { PIK3CA, MYC, BCL2, LPAR1, AKT1, } \\
\text { PIK3R1, IL6R }\end{array}$ & $5.77 \mathrm{E}-08$ & $1.62 \mathrm{E}-04$ \\
\hline $\begin{array}{l}\text { Estrogen signaling } \\
\text { pathway }\end{array}$ & $\begin{array}{l}\text { ATF2, PIK3CA, GNAQ, MMP2, AKT1, } \\
\text { PIK3R1, MMP9 }\end{array}$ & $7.81 \mathrm{E}-07$ & $1.62 \mathrm{E}-04$ \\
\hline Hepatitis B & $\begin{array}{l}\text { IKBKB, ATF2, PIK3CA, MYC, BCL2, } \\
\text { AKT1, PIK3R1, MMP9 }\end{array}$ & $9.40 \mathrm{E}-07$ & $1.62 \mathrm{E}-04$ \\
\hline Pathways in cancer & $\begin{array}{l}\text { IKBKB, PIK3CA, GNAQ, MMP2, } \\
\text { MYC, BCL2, LPAR1, AKT1, BRAF, } \\
\text { PIK3R1, MMP9 }\end{array}$ & $1.98 \mathrm{E}-05$ & $2.39 \mathrm{E}-04$ \\
\hline Colorectal cancer & $\begin{array}{l}\text { PIK3CA, MYC, BCL2, AKT1, BRAF, } \\
\text { PIK3R1 }\end{array}$ & $1.99 \mathrm{E}-05$ & $2.39 \mathrm{E}-04$ \\
\hline $\begin{array}{l}\text { AMPK signaling } \\
\text { pathway }\end{array}$ & $\begin{array}{l}\text { STK11, PRKAA1, CAB39, RAB14, } \\
\text { PIK3CA, AKT1, PIK3R1 }\end{array}$ & $2.99 \mathrm{E}-05$ & $3.14 \mathrm{E}-04$ \\
\hline FoxO signaling pathway & $\begin{array}{l}\text { IKBKB, STK11, PRKAA1, PIK3CA, } \\
\text { AKT1, BRAF, PIK3R1 }\end{array}$ & $4.70 \mathrm{E}-05$ & $3.95 \mathrm{E}-04$ \\
\hline $\begin{array}{l}\text { Signaling pathways } \\
\text { regulating pluripotency } \\
\text { of stem cells }\end{array}$ & $\begin{array}{l}\text { PIK3CA, PCGF5, MYC, AKT1, } \\
\text { PIK3R1, BMI1 }\end{array}$ & $6.58 \mathrm{E}-05$ & $5.02 \mathrm{E}-04$ \\
\hline $\begin{array}{l}\text { Non-alcoholic fatty liver } \\
\text { disease (NAFLD) }\end{array}$ & $\begin{array}{l}\text { IKBKB, PRKAA1, PIK3CA, UQCRQ, } \\
\text { AKT1, PIK3R1, IL6R }\end{array}$ & $1.39 \mathrm{E}-04$ & $7.81 \mathrm{E}-04$ \\
\hline TNF signaling pathway & $\begin{array}{l}\text { IKBKB, ATF2, PIK3CA, AKT1, } \\
\text { PIK3R1, MMP9 }\end{array}$ & $2.04 \mathrm{E}-04$ & $1.07 \mathrm{E}-03$ \\
\hline Insulin resistance & $\begin{array}{l}\text { IKBKB, PRKAA1, PIK3CA, GFPT1, } \\
\text { AKT1, PIK3R1 }\end{array}$ & $2.32 \mathrm{E}-04$ & $1.15 \mathrm{E}-03$ \\
\hline
\end{tabular}

FDR: false discovery rate 


\section{Figure 1}

Figure 1 Patterns of miRNA expression.

(A) Analysis of circulating miRNA expression combined with clinical measures and qRT-PCR data in response to vitamin C supplementation in T2DM subjects with poor glycemic control $(n=5)$. For the miRNA, black and red bubbles represent pre-and post-supplementation, respectively. The size of the bubbles reflects the count of the specific miRNA relative to other miRNAs. The laboratory measures and miR-451a qRT-PCR data are indicated as a heat map from minimum value to maximum value of each parameter independently of other parameters; yellow to blue represents the low to high range. Pre; pre-vitamin C, Post; postvitamin C supplementation. Numbers represent subjects 1-5. (B) Plot represents relative expression of miR-451a as validated by qRT-PCR $(n=8)$.

$\mathbf{A}$

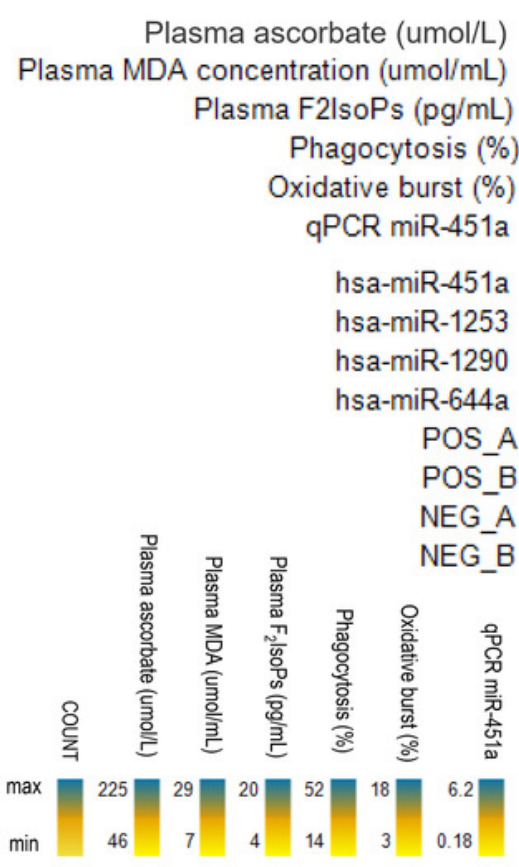

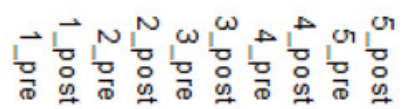

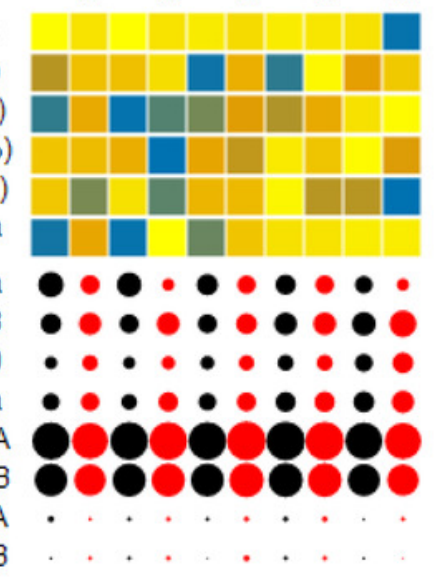
(1)
B

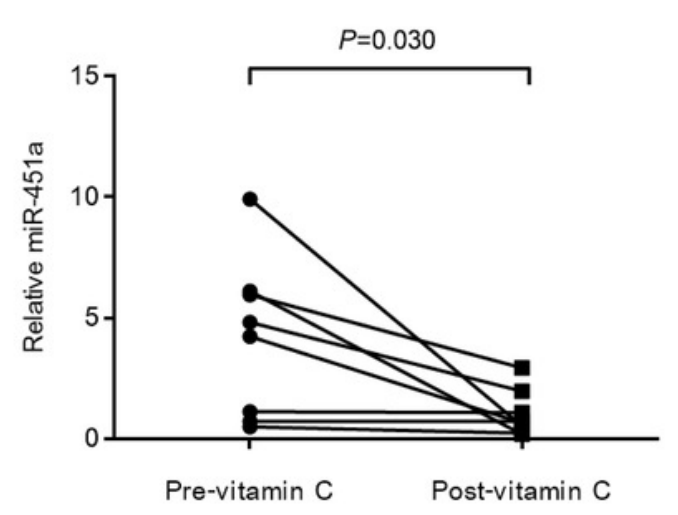


Figure 2

Figure 2 Correlation between miR-451a expression and laboratory variables.

(A) Vitamin C levels, (B) MDA, (C) Cholesterol and (D) LDL. Black and red represent pre-and post-supplementation samples, respectively.

A

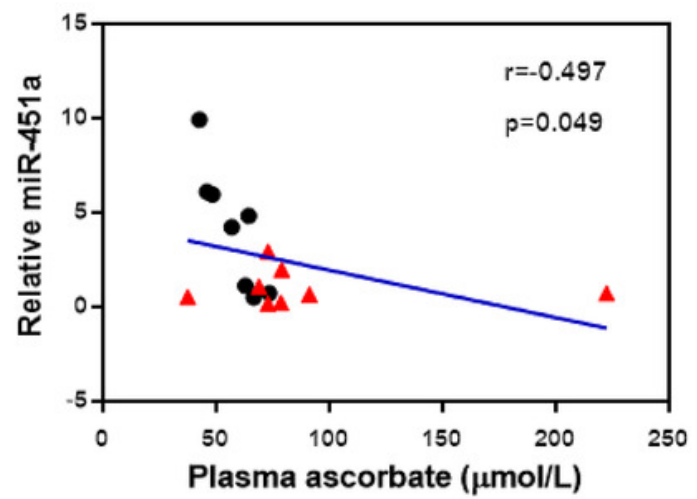

C

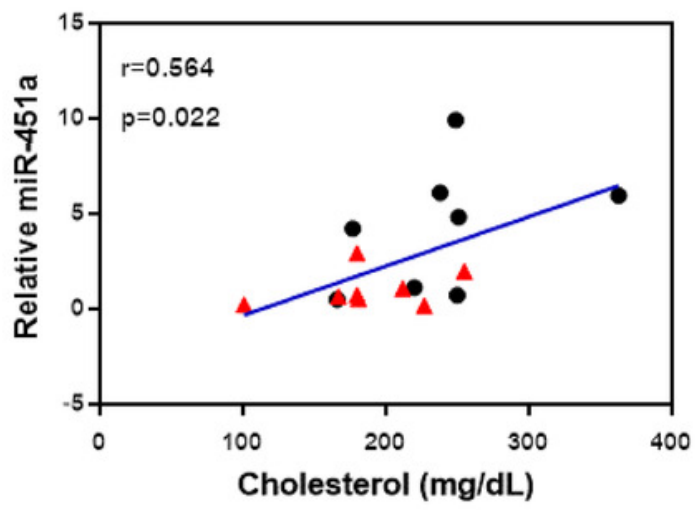

B

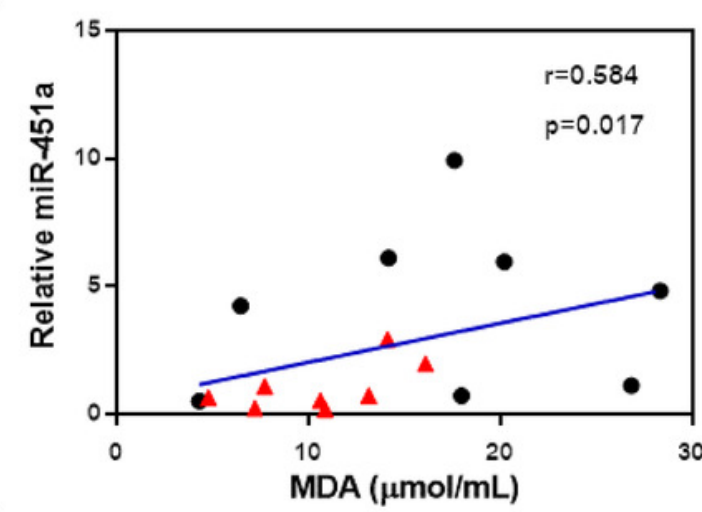

D

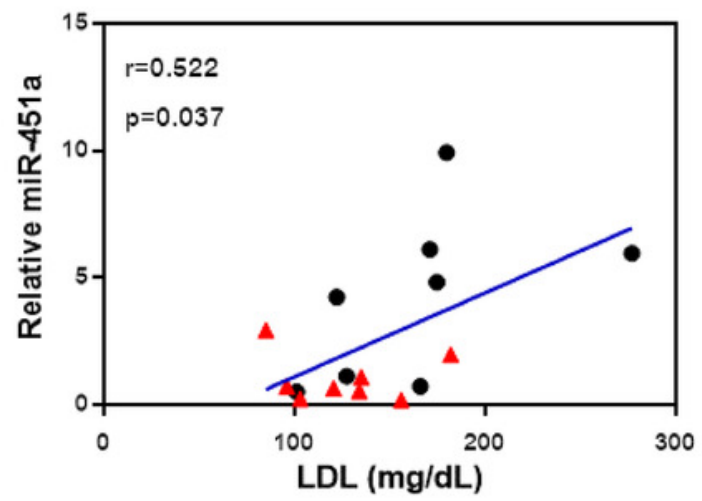

Post-supplementation 


\section{Figure 3}

Figure 3 Proposed mechanism of the effect of miR-451a in response to vitamin C supplementation.

Red lines represent the results of this study and blue lines indicate potential mode of interaction for miR-451a. Poor glycemic controlled T2DM subjects receiving vitamin C 1,000 mg daily for six weeks showed significant down-regulation of circulating miR-451a accompanied with increased levels of vitamin $\mathrm{C}$, reduced oxidative stress (MDA and $\mathrm{F}_{2}$ IsoPs) and increased PMN function (phagocytosis and oxidative burst). Changes in oxidative status may modulate miR-451a expression by altering Ago2 protein and/or increased involvement of signaling pathways such as AMPK signaling by repressing its target genes. MiR-451a may play a role in neutrophil chemotaxis by targeting RAB5A and 14-3-3zeta resulting in the activation of p38 MAPK signaling. However, the role of this miRNA in phagocytosis and oxidative burst of neutrophils is still to be explored (dotted line) and may be reflected in intracellular miRNA changes rather than circulating changes as identified in this study.

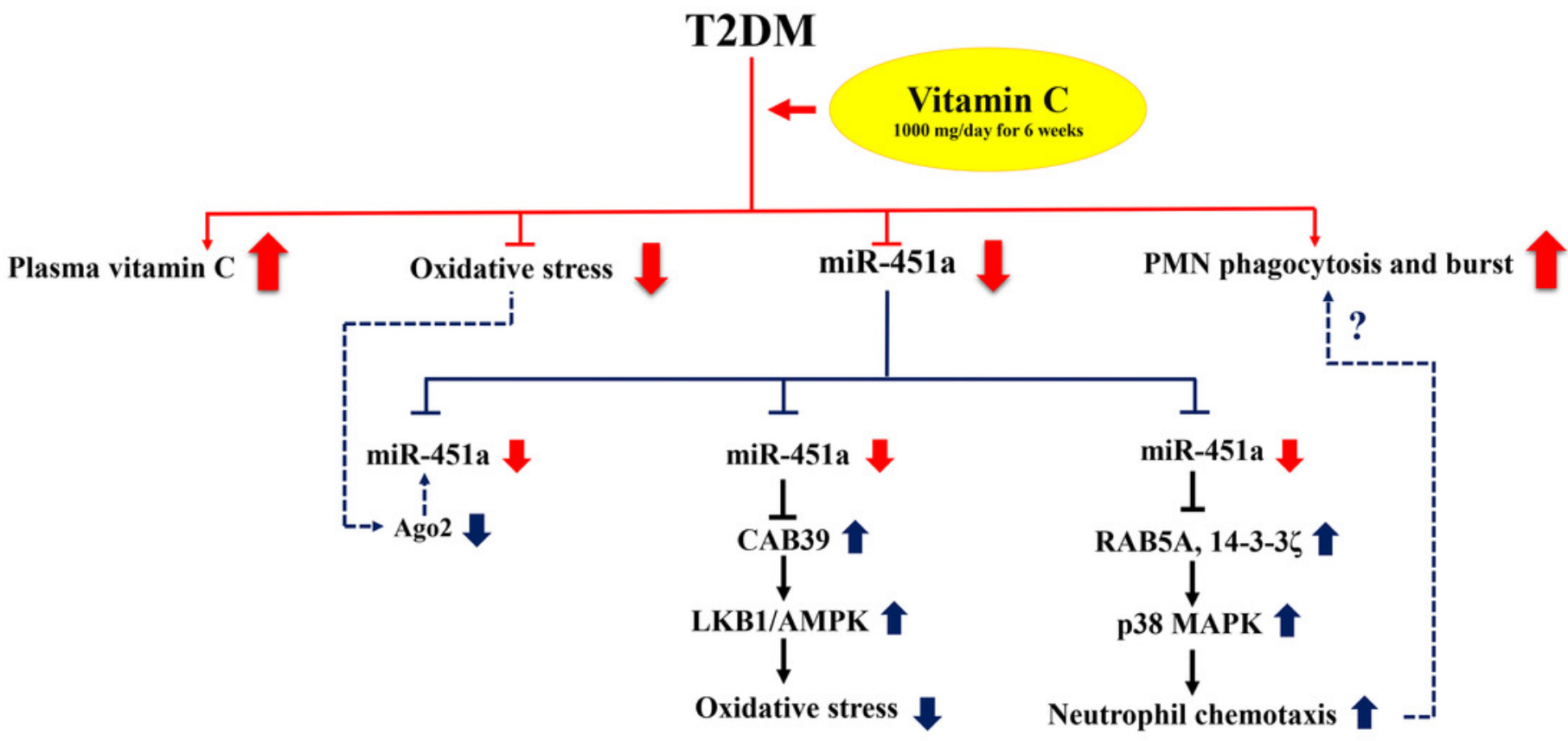

